\title{
Investigating Temporal-Spatial Characteristics of Mouse and Touch Input
}

\author{
Christian Müller-Tomfelde \\ CSIRO ICT Centre, \\ Cnr Vimiera and Pembroke Roads, \\ Marsfield NSW 2122, Australia \\ Christian.Mueller-Tomfelde@csiro.au
}

\begin{abstract}
This paper explores differences between user actions employing mouse and touch input devices when performing a collaborative task on a tabletop interface. We explore temporal and spatial characteristics of drag actions of users through mouse (indirect) and touch input (direct) devices. Our analysis is based on system-logged interaction data we collected in an exploratory study. The analysis reveals that touch users performed more drag actions than mouse users. Furthermore, touch users dragged artefacts on a tabletop for a shorter period of time than mouse users. At the same time, touch users dragged objects in the workspace shorter distances than mouse users. We also identify differences based on the temporal-spatial histograms of the drag actions. We discuss our findings based on observations we made during the collaborative task and interviews with participants after the study. The results may be related to the performed task and to ergonomic design issues of the setup. We describe the possible implications on co-located and remote collaboration.
\end{abstract}

Keywords: Direct and indirect input devices, synchronous co-located collaboration, tabletop, user study.

\section{Introduction}

Tabletop environments providing touch interaction are popular settings to support the collaborative work of co-located small teams $[1,2,3,4]$. While the benefits of touch input devices for tabletops have been identified [5,6], studies have been conducted to further understand the differences of touch and mouse input devices on individual task performance [7] and the implications of different interaction techniques on collaboration on tabletops [8]. Advantages and disadvantages have been documented to provide guidelines for design decisions for co-located collaboration at tabletops [5]. Differences between touch and mouse interactions have also been studied for unimanual and bimanual tasks of individuals [9]. The authors report the benefits for direct touch interaction for bimanual tasks and indicate that mouse devices might be more appropriate for individuals working on so called standard "single-point" interaction tasks.

We conducted an exploratory study on input device preferences and usage during co-located collaboration. The study revealed a balanced preference for mouse and 
touch as input devices for the particular tabletop setup [10]. The system-logged interaction data of this exploratory study forms the basis of the detailed analysis in this paper. The contribution of this paper is twofold: firstly, we provide further insights to the characteristics of the temporal-spatial organisation of drag actions based on mouse and touch as input device during the collaborative task. These characteristics between mouse and touch users differs, despite the fact that there was no main effect of the input device on the task completion. Secondly, we discuss these findings with respect to observations made during the trials and to the particularities of the experimental setup. We discuss possible implications of our findings in the context of co-located and remote collaboration on tabletops.

\section{Collaboration Study}

In our exploratory study 22 participants (in 11 trials) were asked to solve jigsaw puzzle tasks with increasing assembly complexity. The study emphasises a quasi-realistic context with two work colleagues standing side by side at a tabletop to work in parallel (see Figure 1).

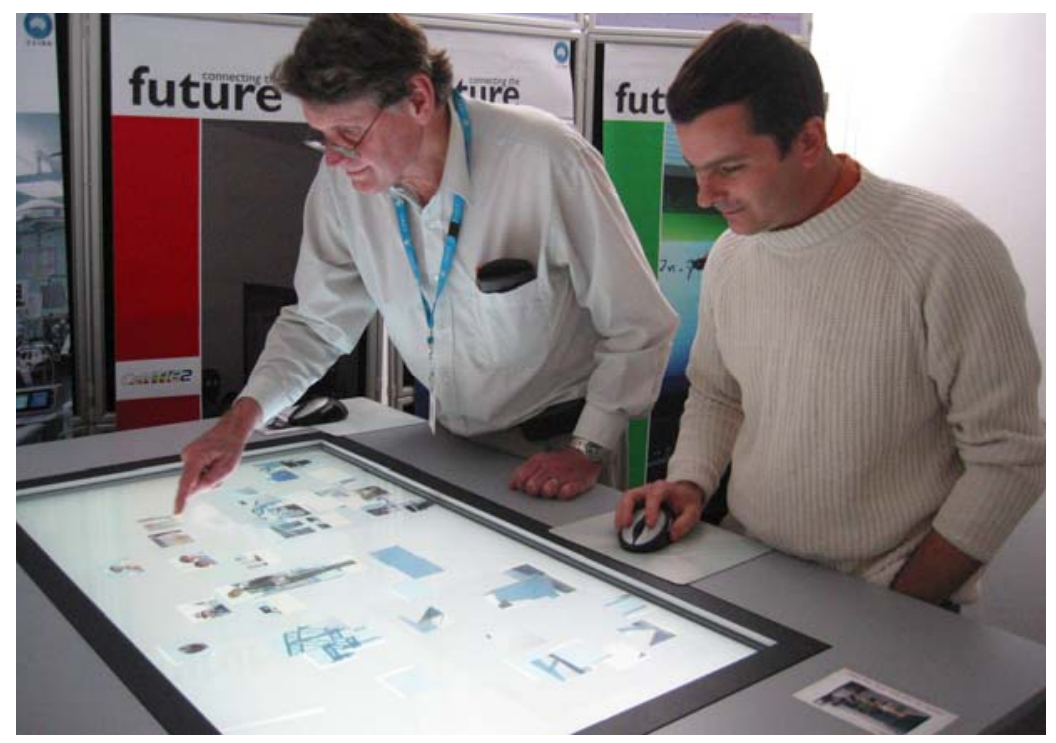

Fig. 1. In the exploratory study, participants were standing side-by-side using either mouse or touch input device to collaborate

More details about the setup are described in [10, 11]. The participants' task was to drag puzzle pieces on the tabletop surface to assemble several photo pictures. Both participants had to each solve one puzzle in front of them. The interface was designed to mimic that of real jigsaw puzzles or mosaics, except that it did not allow for rotation, lifting up or any sort of tangibility of the pieces. Solving one puzzle task lasted in average $7 \mathrm{~min}$. In contrast to other studies (e.g. $[9,6])$, we granted the participants 
the free choice to select and change their input device at any time during the study. As a consequence the input device in this study has to be considered as a dependent variable rather than an independent one. The experimental task was then increased in difficulty by mixing pieces between the two puzzles. This was implemented to trigger spontaneous coordination and communication amongst the team members as well as to find out how this might affect the usage of the input devices. The spatial layout of the physical task space may give the impression that each participant owned one puzzle and that individuals worked in parallel. However in all of the trials the participants worked collaboratively on the task, i.e., they communicated, observed each other workspaces and coordinated actions.

The goal of our study was to achieve, as much as possible, ecological validity of the task and setting in our lab environment. We collected qualitative and quantitative data of the participants' interactions through observations, and objective measures in the form of system-logged interaction data. The focus of this paper is on the objective measures and related observations to further understand the difference between mouse and touch device users in this tabletop setting.

\section{Manipulating the Artefact}

In order to further understand differences between the usage of direct and indirect input devices we explore the participants' behaviour at an input device level. We are focusing on drag actions, which we define as the action of a user dragging puzzles pieces on the tabletop. For instance, a mouse user can move the cursor on the screen at any time, but only drags an object when moving it after selecting the object by pressing the mouse button down. The period of moving the cursor towards a target object until pressing the mouse button is usually described using Fitts' law [12] (selection time). In contrast to this, we are focusing on the continuous drag actions after having selected the object and before releasing the object at a target position (similar to the docking task described in [9]). In the case of our touch input device, the position and movement of the finger could only be captured when it was in close proximity to the surface. Therefore, the selection time becomes difficult to compare between the devices and we concentrate on the manipulation of the artefact based on drag actions. It should be noted that the recorded drag actions are self-paced, as in [8, 13], rather than stimulus driven as in experiments, such as [9]. For our analysis, we generated the two measures, drag time and drag distance, from the interaction data.

\subsection{Drag Time and Distance}

We define drag time as the period of time of a drag action, i.e., the duration from the selection of a jigsaw puzzle piece by touch or mouse click until its release at a final position. The drag time starts with the selection of a puzzle piece, followed by its translation on the surface. The drag time stops with the release of the object at the final position. From the coordinates of a drag action we derive a distance measure. In a fist approach, we define the drag distance as the L2-norm of the coordinate vector from the start to the end point of a drag action. Other distance measures, e.g., along the trajectory of the path of action, might be useful to investigate in the future. 


\subsection{Collection of Interaction Data}

We developed customised software to support up to four input devices (i.e., dual touch plus two mice) to concurrently control graphical elements for the experiment. Our application recorded all interactions with attributes such as time, device ID, position, state (e.g., move, drag), and events (e.g., button down or up). Based on this data we derived the overall time to complete the task and the period of time that participants actually drag puzzle pieces around. Further refinement of the interaction data allowed us to access all drag actions per interaction device.

\section{Analysis}

Based on the interaction data of 11 trials $(\mathrm{N}=22)$ we processed all drag actions with regard to their times, distances and input device type. The participants could be indentified as purely touch or mouse users, except in one trial where the two participants swapped the input device type during the trial. We excluded this trial to have an unequivocal classification. Thus, our results base on 10 trials:

- 2962 drag actions by mouse (9 participants) and

- 5497 drag actions by touch input device (11 participants).

We recorded 1.85 more drag actions with touch than with mouse input device. The average number of drag actions per participant was approximately 500 for direct and 330 for indirect input devices. The descriptive statistics for drag times and distances categorised by input device are shown in Table 1.

Table 1. Descriptive statistics of the drag times and drag distances of direct and indirect input devices

\begin{tabular}{|c|c|c|c|c|c|c|}
\hline $\begin{array}{c}\text { Device } \\
\text { Type }\end{array}$ & Min & $\begin{array}{l}1^{\text {st }} \\
\text { Qu. }\end{array}$ & Median & Mean & $\begin{array}{l}3^{\text {rd }} \\
\text { Qu. }\end{array}$ & $\operatorname{Max}$ \\
\hline & \multicolumn{6}{|c|}{ Drag time [s] } \\
\hline Indirect & .078 & .832 & 1.500 & 2.028 & 2.5630 & 93.610 \\
\hline \multirow[t]{2}{*}{ Direct } & .062 & .438 & 0.875 & 1.161 & 1.578 & 11.420 \\
\hline & \multicolumn{6}{|c|}{ Drag distance $[\mathrm{cm}]$} \\
\hline Indirect & .048 & 4.312 & 11.72 & 15.05 & 23.69 & 86.71 \\
\hline Direct & .048 & 2.103 & 6.323 & 10.38 & 15.53 & 80.29 \\
\hline
\end{tabular}

For both input device types the extreme small time values below $200 \mathrm{~ms}$ are likely be caused by artefacts of the detection. Manipulations with such a short execution time can not be considered to be deliberately executed actions [14] and were not used for this analysis. We can state that the median value of the drag times with mouse input device is approximately 0.5 seconds higher than that for touch. The median value of the mouse drag distances is approximately twice the value of that of touch. 


\section{Drag Time Histograms}

The histograms of the drag times are shown in Figure $2 \mathrm{a}$ and $2 \mathrm{~b}$. The histograms are both skewed to the right, similar to those of human reaction time histograms. Histograms for the drag distances report a similar but slightly less positively skewed distribution. We must assume that the drag times and distances are not normally distributed and hence parametric significance tests such as t-tests are not applicable. A Wilcoxon rank-sum test of the drag times and drag distances between direct and indirect input device reveal significant differences (each $\mathrm{p}<0.001)$ in the locations.

a)

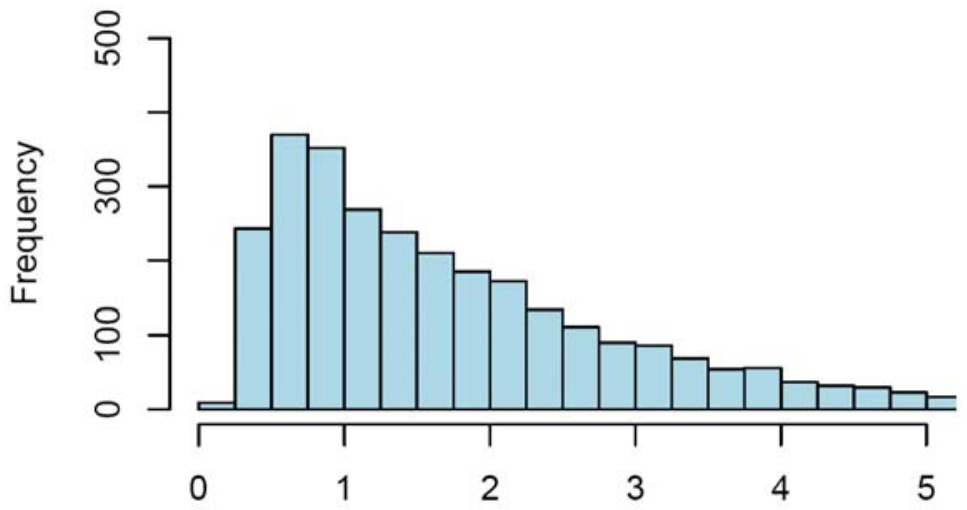

drag time [s]

b)

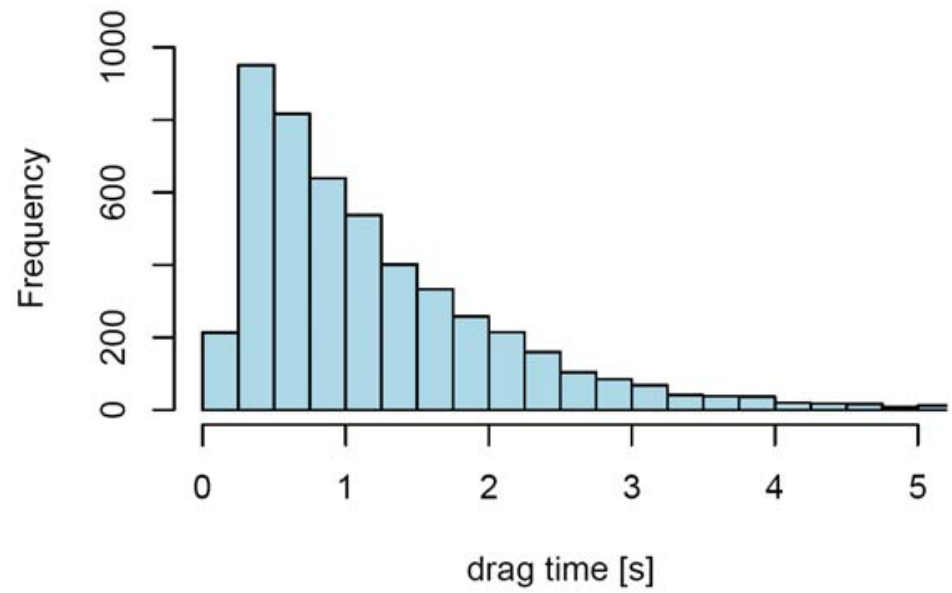

Fig. 2. Histograms of the drag times using either mouse or touch input device. Graph a) shows the histogram for indirect device (mouse) and b) the histogram for direct device (touch).

\section{Temporal-Spatial Histograms}

To understand more about the relationship between drag time and drag distance we plotted two-dimensional histograms (see Figure 3). These histograms show the distribution of drag actions of mouse and touch users in the temporal-spatial domain. 
a)
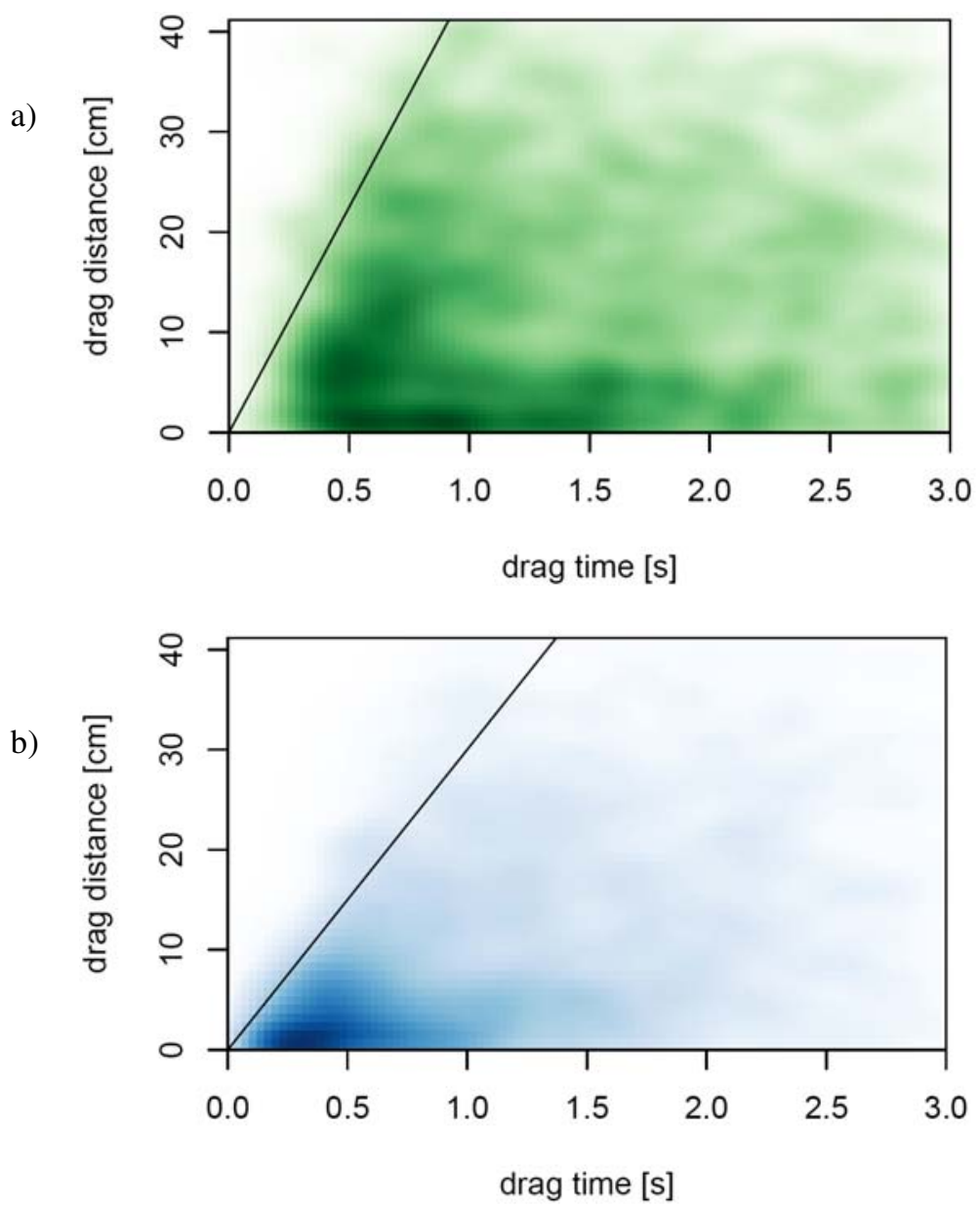

Fig. 3. Temporal-spatial histograms of a) indirect input (mouse) device drag actions and b) direct device (touch) drag actions. The lines represent the speed limits of drag actions.

Darker shaded areas have a higher frequency than lighter shaded areas. White areas signify minor or no occurrence of drag actions. Each histogram shows a distinct maximal drag speed of approximately $45 \mathrm{~cm} / \mathrm{s}$ for indirect (represented by the line in Figure $3 \mathrm{a}$ ) and $30 \mathrm{~cm} / \mathrm{s}$ for direct input devices (Figure $3 \mathrm{~b}$ ).

Furthermore, the histogram of the indirect input device (Figure 3a) is more distributed in the temporal-spatial space than that of the direct input device. In particular, the histogram in Figure 3b (direct input) is much more focused on the area around and below 0.5 seconds with distances less than $7.5 \mathrm{~cm}$. In contrast, the histogram of the indirect input (Figure 3a) has temporally long lasting drag actions with relatively short distances (less than $7.5 \mathrm{~cm})$. Also many temporally short drag actions between $(0.5$ and $1 \mathrm{~s}$ ) over a long distance, i.e., with high drag speed occurred. We also plotted the all drag actions of mouse and touch users in the two dimensional plain of the workspace to 
identify possible specific spatial patterns. However, we can report that the spatial organisation of drag actions of mouse and touch had no apparent differences.

\section{Observations}

We observed that all participants worked without any problems or complains on the task with either input device. Even the coordination of actions of both participants, i.e., the handing over of puzzle pieces from one to the other happened smoothly and without additional training $[15,16]$. All participants concentrated and cooperated on the task and they shared their effort even more and developed strategies when they found out that puzzle pieces were mixed up (second sub trial). The communication amongst them increased as well as their gazes on the other's work area and number of coordinated actions.

Reviewing the video recordings of the trials we can report that the participants using the mouse exhibit clearly fewer physical body movements than touch users. The latter moved most of them time the arm of their dominant hand to manipulate the artefact in front of them on the display. Mouse users seem to remain still, only moving their hand resting on the rim of the table controlling the mouse (see Figure 1). Touch and mouse users moved their heads to follow the currently dragged puzzle piece or to better oversee the display seeking of a possible matching piece. Touch device users seem to be much more active. In trails with one mouse and one touch device user it appeared to the observers that only the touch user was active although they both were working highly concentrated on the task. The reduced physical body activity of participants using mouse devices comes also along with less physical effort during the task. Eventually all participants changed their general posture at the table, e.g., balancing their weight from on foot to the other. The participant however did not complained about standing at the table during the task.

We interviewed the participants after they completed the study. Participants who used direct touch input devices reported an almost similar experience as with a real puzzle (e.g., P1, trial 1). A touch user stated that "the touch is much more human" (P2, trial 5). One participant reported that with the touch device "it was a lot easier to get control" over the puzzle piece (P2, trial 7). Some mouse users were confident to work faster with the mouse than with the touch device (P2, trail 3) and P1 in trial 6 explained his input device preference that he "... uses [the] mouse mostly". One participant reported an ergonomic issue using the mouse without elbow support (P1, trial 3).

Both participants in trial 7 reported that they were not tempted to use the mouse even when they had to reach far away objects. This was also mentioned by P1 (trail 5) "it just didn't occurred to me". We observed however that some participants spontaneously changed temporally the input device but continue to work with their preferred input device after only a few manipulations. None of the participants mentioned that physical effort was an issue during the task. It was also not mentioned to be reason to choose one input device before the other.

We observed also a particular class of drag actions. Some of the participants acted beyond the instructed task and arranged the jigsaw pieces in the final stage of the task with high positioning precision. This happened, although the experimenters informed the participants at the beginning of the trial that precision does not matter for solving 
this task. This behaviour of "beautifying" the result of the work could be considered different to a normal drag action and might need to be removed from the data. While normal drag actions are carried out to reach the communicated goal of the experiment task, these beautifying actions serve more of an individual and aesthetic goal or desire. We observed that while these actions have short drag distances, they can also have long drag times. However, the impact on the findings are considered to minor, due to the fact that these particular actions happened only occasionally and had been carried out by users of both types of input device.

\section{Discussion}

We assume that our setting is typical for tabletop studies and that the task and collaboration are ecologically valid. However, the above described findings and observations strictly hold only for our specific setting of the study, such as the side-by-side position arrangement (other arrangements can be considered [17]). The presented quantitative differences in drag times and distances of touch and mouse input need to be discussed in the context of our observations and qualitative statements of the interviews of the participants. We discuss how the specific properties of the input devices might shape the way drag actions are carried out differently by the participants using an indirect or direct input device. Furthermore, the constructs of epistemic actions and complementary strategies as proposed by Kirsh [18, 19] might help to explain our findings and observations of the collaborative task.

\subsection{Input Device Ergonomics}

There are different ergonomic aspects of our experimental setting as depicted in Figure 1. We concentrate on the ergonomics of the input devices and assume that other aspects such as the posture and position arrangements of the participants have only indirect or minor influence on the reported findings. For instance, the standing posture just gives the touch users a larger area to act in (see [20]) and better visibility of the workspace. Our observations reveal that touch users require more physical effort than mouse users when reaching pieces and moving them around. Since the participants using the touch input devices never mentioned or even complained about the physical effort we must assume that this extra physical effort compared to those of mouse users is not apparent to the participants. Mouser users remained nearly motion less merely performing actions controlling the mouse and occasionally turning their heads slightly overseeing the partners' workspaces. Thus, their activity and intentions are less likely to become recognised by their partners than those of touch users. This provides plausibility for the fact that mouse users dragged pieces for a longer time because only small changes in the hand position are required. Instead, touch users performed drag actions confined to a particular region in the temporal-spatial histogram (see Figure 3b). Twice the number of the drag actions with touch than with mouse might compensate for these differences in temporal-spatial histogram of the drag actions. However it seems that the extra physical effort does not have an impact on the efficiency working on the task. We found no significant difference in the overall task completion time depending on the input device [10]. 


\subsection{Epistemic Actions}

Most of the participants had reported to have experience with solving jigsaw puzzles, and therefore it is likely that they pursued already established strategies to solve the puzzle task. Typical to this task is that users perform a visual search task that is accompanied and combined with so called "epistemic actions". This class of action is preformed to "uncover information that is hidden or hard to compute mentally" and therefore "change[s] the world in order to simplify the problem-solving task" [19]. In our example a typical epistemic action would be moving a puzzle piece towards potential matching pieces. By doing so, the visual search and the decision about whether two piece are matching or not becomes simplified. In case of a positive match the epistemic action becomes seamlessly transformed into a "pragmatic action". This action is executed directly to accomplish the goal of the task. Also "complementary strategies" might be exhibited, i.e., "any organizing activity which recruits external elements to reduce cognitive loads" [18]. The participants build subgroups of pieces with similar content or colour to structure and simplify process of solving the puzzle.

It has to be discussed how these actions and strategies become support or hindered by the digital artefact itself and by the different input device technologies. The fact that the puzzle pieces are displayed on the screen "only" and lack of any tangibility reduce the traditional handling of the pieces, e.g., with full hands. Therefore actions or strategy that requires full hand or bimanual interaction were impossible to perform in this setting. For instance, it is obvious that a pile of puzzle pieces can not simply distributed by just shuffling multiple pieces with a flat hand. Also a group of pieces can not be moved as a set to another location. Instead all pieces had to be moved individually with touch as well as with mouse input device.

However, the different input devices might support or inhibit epistemic actions and complementary strategies that led to our finding. We are focussing on the epistemic action of finding a matching piece by dragging it around. Our analysis of the drag actions suggests that it is possible that mouse users exhibited more of these epistemic actions than touch users. Mouse users not only drag objects longer but also show greater variety in the temporal-spatial distribution. It seems likely that persons using the mouse pick up a piece without having decided in advance where to place it due to the low physical effort required. In contrast touch user are to more consistent in their action (see Figure 3b) which suggests that they rather perform the drag action after they decided where to place the piece. Thus touch user minimise their physical effort and maximising efficiency of the drag actions. Instead of performing one long drag action in search for a match touch, users might also break up a search for a match into a sequence of short drag actions.

The key of the extension of drag actions in time and space with the mouse beyond those of touch actions however may lie in the very little physical effort that is required to manipulate the artefact with the mouse, similar to the effort in tradition desktop computer settings. We suggest that the different physical effort required by the specific input device to manipulate the artefact has an impact on the quality of the users' actions while solving the puzzle task. If an input device demands little physical effort more epistemic actions are likely to be performed. 


\subsection{Implications on the Collaboration}

So far we discussed the difference of the drag actions for the individual performance ignoring the possible impact on the collaboration. We discuss the implication of our findings on the collaboration using the concept of workspace awareness and embodiment of actions. Workspace awareness is the understanding of another person's actions and "includes knowledge where and on what others are currently working and what they are doing next" [21]. It is supposed that workspace awareness supports the coordination during collaboration. Our findings allow us to conclude that touch device user provide more workspace awareness to their partners than mouse users. The shear amount of physical actions of touch users with theirs hands and arms provide richer peripheral awareness for their partners than to mouse users do. These physical actions show if and where the partner is working, and provides very well the information which puzzle piece is currently in focus. Recent findings also comparing touch and mouse actions presented in [22] support this conclusion. Other research comes also to similar conclusion comparing different interaction techniques [8]. The richer peripheral awareness created by touch users comes long with possible workspace occlusion and physical conflicts in case of multiple touch users actions in the same workspace area.

However when only focussing only changes in the workspaces created by drag actions another aspect of our findings comes into play. We consider now an environment, where two spatially distributed setting as used in our study form a shared workspace. This would lead to a similar environment as used in $[23,13]$ but without the rich remote action embodiments such as arm shadow [24]. The temporally longer drag actions in this distributed collaboration scenario could facilitate the communication and coordination better than touch. The longer drag actions provide more workspace awareness for remote collaborators since they can perceive more changes in the workspace. Our findings also show that drag actions with the mouse device have greater temporal-spatial variety than those of touch device users. This fact may imply that also more different classes of actions are performed. As argued in the section before, the particular epistemic actions with the mouse have the potential to better support awareness since they contain and convey to the remote site more meaning or intentionality than the touch actions.

To sum up the implication on collaboration, we can draw the following conclusion. In a straight comparison between the devices, the touch device is highly beneficial for co-located collaboration due to the natural and rich awareness of the users' physical actions with their arms and hands. In contrast, the mouse device bears the potential to better provide awareness in the collaboration with a remote partner because more meaningful changes in the workspace occur. An interesting research topic emerges here when developing an environment that combines the benefits of both input devices for distributed collaboration, i.e., when local mouse actions become remotely embodied with richer embodiments than standard mouse cursors.

\section{Conclusion}

We presented results from a detailed analysis of drag times and drag distances of touch and mouse interaction data collected during an earlier study. The analysis of 
these measures shows that touch users drag objects for shorter periods of time over shorter distances than mouse users. The temporal-spatial histograms of the drag actions reveal further characteristics of the specific input device. Mouse drag actions have greater variety in time and distance while touch drag actions are more focussed. These findings go beyond the existing knowledge about the characteristics of direct and indirect input device interaction. We discussed the finding in the context of the ergonomics of the input devices and of epistemic actions. The findings could be useful when developing new interaction techniques for distributed collaboration.

\section{Acknowledgements}

This research was conducted within the HxI Initiative [25], an Australian research initiative led by CSIRO, DSTO and NICTA [26]. The author thanks the test participants for allowing us to collect and publish the presented data. I also thank Claudia Schremmer in helping, organising and conducting the study and Anja Wessels for helping with the hands-on running of the experiment and the collection of experiment data.

\section{References}

1. Rauterberg, M., Fjeld, M., Krueger, H., Bichsel, M., Leonhardt, U., Meier, M.: BUILD-IT: a planning tool for construction and design. In: Proceedings of the SIGCHI conference on Human factors in computing systems (CHI 1998), pp. 177-178. ACM Press, New York (1998)

2. Rekimoto, J., Saitoh, M.: Augmented surfaces: a spatially continuous work space for hybrid computing environments. In: Proceedings of the SIGCHI conference on Human factors in computing systems (CHI 1999), pp. 378-385. ACM Press, New York (1999)

3. Streitz, N.A., Geißler, J., Holmer, T., Konomi, S., Müller-Tomfelde, C., Reischl, W., Rexroth, P., Seitz, P., Steinmetz, R.: i-LAND: an interactive landscape for creativity and innovation. In: Proceedings of the SIGCHI conference on Human factors in computing systems (CHI 1999), May 15-20, pp. 120-127. ACM Press, New York (1999)

4. Shen, C., Everitt, K., Ryall, K.: UbiTable: Impromptu face-to-face collaboration on horizontal interactive surfaces. In: Dey, A.K., Schmidt, A., McCarthy, J.F. (eds.) UbiComp 2003. LNCS, vol. 2864, pp. 281-288. Springer, Heidelberg (2003)

5. Scott, S.D., Grant, K.D., Mandryk, R.L.: System guidelines for co-located, collaborative work on a tabletop display. In: Proceedings of the eighth conference on European Conference on Computer Supported Cooperative Work (ECSCW 2003), Norwell, MA, USA, pp. 159-178. Kluwer Academic Publishers, Dordrecht (2003)

6. Ha, V., Inkpen, K., Mandryk, R., Whalen, T.: Direct intentions: the effects of input devices on collaboration around a tabletop display. In: Proceedings of the First IEEE International Workshop on Horizontal Interactive Human-Computer Systems (TABLETOP 2006), Adelaide, Australia, January 5-7, pp. 175-190. IEEE Computer Society, Los Alamitos (2006)

7. Huang, C.J.: Not just intuitive: examining the basic manipulation of tangible user interfaces. In: CHI 2004 extended abstracts on Human factors in computing systems (CHI 2004), pp. 1387-1390. ACM Press, New York (2004) 
8. Nacenta, M.A., Pinelle, D., Stuckel, D., Gutwin, C.: The effects of interaction technique on coordination in tabletop groupware. In: Proceedings of Graphics Interface 2007 (GI 2007), pp. 191-198. ACM Press, New York (2007)

9. Forlines, C., Wigdor, D., Shen, C., Balakrishnan, R.: Direct-touch vs. mouse input for tabletop displays. In: Proceedings of the SIGCHI conference on Human factors in computing systems (CHI 2007), pp. 647-656. ACM Press, New York (2007)

10. Müller-Tomfelde, C., Schremmer, C., Wessels, A.: Exploratory study on concurrent interaction in co-located collaboration. In: Proceedings of the 2007 conference of the computerhuman interaction special interest group (CHISIG) of Australia on Computer-human interaction: design: activities, artifacts and environments (OZCHI 2007), pp. 175-178. ACM Press, New York (2007)

11. Müller-Tomfelde, C.: Interacting with mouse and touch devices on horizontal interactive displays. International Journal Universal Access in the Information Society (in press, 2009)

12. Fitts, P.: The information capacity of the human motor system in controlling the amplitude of movement. Experimental Psychology, 381-391 (1997)

13. Pauchet, A., Coldefy, F., Lefebvre, L., Louis Dit Picard, S., Perron, L., Bouguet, A., Collobert, M., Guerin, J., Corvaisier, D.: TableTops: worthwhile experiences of collocated and remote collaboration. In: Proceedings of the Second Annual IEEE International Workshop on Horizontal Interactive Human-Computer Systems (TABLETOP 2007), Newport, Rhode Island, USA, October 10-12, pp. 27-34. IEEE Computer Society Press, Los Alamitos (2007)

14. Card, S., Moran, T., Newell, A.: The Psychology of Human-Computer Interaction. Lawrence Erlbaum, Hillsdale (1983)

15. Jun, L., Pinelle, D., Gutwin, C., Subramanian, S.: Improving digital handoff in shared tabletop workspaces. In: Proceedings of the 3rd IEEE International Workshop on Horizontal Interactive Human Computer Systems (TABLETOP 2008), October 1-3, pp. 9-16 (2008)

16. Müller-Tomfelde, C., Schremmer, C.: Touchers and Mousers: commonalities and differences in co-located collaboration with multiple input devices. In: Proceeding of the twenty-sixth annual SIGCHI conference on Human factors in computing systems (CHI 2008), pp. 1149-1152. ACM Press, New York (2008)

17. Tang, A., Tory, M., Po, B., Neumann, P., Carpendale, S.: Collaborative coupling over tabletop displays. In: Proceedings of the SIGCHI conference on Human Factors in computing systems (CHI 2006), pp. 1181-1190. ACM Press, New York (2006)

18. Kirsh, D.: Complementary strategies: why we use our hands when we think. In: Proceedings of the Conference of the Cognitive Science Society, Hillsdale, New Jersey. Lawrence Erlbaum, Mahwah (1995),

http://www. cogsci.ucsd.edu/ kirsh/Cogsci95/cogsci95.html (cited 22.01.2009)

19. Kirsh, D., Maglio, P.: On distinguishing epistemic from pragmatic action. Journal on Cognitive Science, 513-549 (1994)

20. Toney, A., Thomas, B.H.: Considering reach in tangible and table top design. In: Proceedings of the First IEEE International Workshop on Horizontal Interactive Human-Computer Systems (TABLETOP 2006), Adelaide, Australia, January 5-7, pp. 57-58. IEEE Computer Society Press, Los Alamitos (2006)

21. Gutwin, C., Greenberg, S.: A descriptive framework of workspace awareness for real-time groupware. Computer Supported Cooperative Work (3), 411-446 (2002)

22. Hornecker, E., Marshall, P., Dalton, N.S., Rogers, Y.: Collaboration and interference: awareness with mice or touch input. In: Proceedings of the ACM 2008 conference on Computer supported cooperative work (CSCW 2008), pp. 167-176. ACM Press, New York (2008) 
23. Tuddenham, P., Robinson, P.: Distributed Tabletops: Supporting remote and mixedpresence tabletop collaboration. In: Proceedings of the Second Annual IEEE International Workshop on Horizontal Interactive Human-Computer Systems (TABLETOP 2007), Newport, Rhode Island, USA, October 10-12, 2007, pp. 19-26. IEEE Computer Society Press, Los Alamitos (2007)

24. Tang, A., Neustaedter, C., Greenberg, S.: Videoarms: Embodiments for mixed presence groupware. In: Bryan-Kinns, N., Blanford, A., Curzon, P., Nigay, L. (eds.) Proc. of HCI on People and Computers XX. Springer, Heidelberg (2006)

25. HxI Initiative (2008), http: / /www . hxi . org . au (cited 05.01.2008)

26. Schremmer, C., Müller-Tomfelde, C.: HxI: research down under in distributed intense collaboration between teams. In: CHI 2008 extended abstracts on Human factors in computing systems (CHI 2008), pp. 3645-3650. ACM Press, New York (2008) 